\title{
Hyperuniform density fluctuations and diverging dynamic correlations in periodically driven colloidal suspensions
}

\author{
Elsen Tjhung and Ludovic Berthier \\ Laboratoire Charles Coulomb, UMR 5221, CNRS and Université Montpellier, Montpellier, France
}

(Dated: August 20, 2018)

\begin{abstract}
The emergence of particle irreversibility in periodically driven colloidal suspensions has been interpreted as resulting either from a nonequilibrium phase transition to an absorbing state or from the chaotic nature of particle trajectories. Using a simple model of a driven suspension we show that a nonequilibrium phase transition is accompanied by hyperuniform static density fluctuations in the vicinity of the transition, where we also observe strong dynamic heterogeneities reminiscent of dynamics in glassy materials. We find that single particle dynamics becomes intermittent and strongly non-Fickian, and that collective dynamics becomes spatially correlated over diverging lengthscales. Our results suggest that the two theoretical scenarii can be experimentally discriminated using particle-resolved measurements of standard static and dynamic observables.
\end{abstract}

PACS numbers: 05.40.-a, 05.65.+b, 47.57.E-

Nonequilibrium phase transitions have been studied intensively in recent years [1, 2. Whereas many theoretical models have been analysed and organized in a small number of universality classes (such as directed percolation), convincing experimental realisations have typically proved harder to achieve. Non-Brownian colloidal suspensions (such as stabilized droplet emulsions or large particles suspended in a viscous solvent) driven by a lowfrequency periodic shear flow represent one potential realisation of a second-order phase transition towards an absorbing state [3 9]. It has been found experimentally that below a certain shearing amplitude (which depends on the density), the system evolves after a transient into a reversible state where all particles return to the same position at the end of each cycle of the periodic drive. Above a well-defined threshold amplitude, particle motion are no longer periodic, and a continuous increase of diffusive motion is observed in this irreversible phase 3 .

Several studies 4, 6, 10,13, suggested that the experimental transition is in the universality class of directed percolation (or conserved directed percolation). This interpretation is further supported by an elegant numerical model of the original experiment, which was shown to undergo a second-order nonequilibrium phase transition 4. However, an alternative explanation was also proposed 14 19, which relies on the chaotic nature of trajectories in dynamical systems. In this view, a phase transition is not needed to explain the relatively sharp onset of irreversilibity observed in the experiments. Experiments have not fully established criticality because direct measurements of the critical exponents are difficult $[3,4,13,16$. As a result, the nature of the initial experimental observations remains to be fully understood. Here we establish that measurements based on standard particle-resolved observables developed in the context of glassy dynamics 20] very directly reveal nonequilibrium criticality, when present. This suggests that the two existing theoretical scenarii can be experimentally discri- mated using standard static and dynamic observables.

To support our conclusions, we consider a modified version of the model proposed in Ref. [4], as illustrated in Fig. 1(a). We consider a bidimensional assembly of spherical particles of diameter $\sigma$, using periodic boundary conditions in a box of linear size $L$. The system is initiated from a random configuration, where particle overlaps may exist. At each time step, we simultaneously move all particles which overlap with one neighbor (or several) by an independent random amount. The displacement of particle $i$ is of the form $\vec{\delta}_{i}=\epsilon_{i} \hat{e}_{i}$, where $\hat{e}_{i}$ is a unit vector whose orientation is uniformly distributed on a unit circle and the magnitude $\epsilon_{i}$ is uniformly distributed on the interval $[0, \delta]$. The time is then
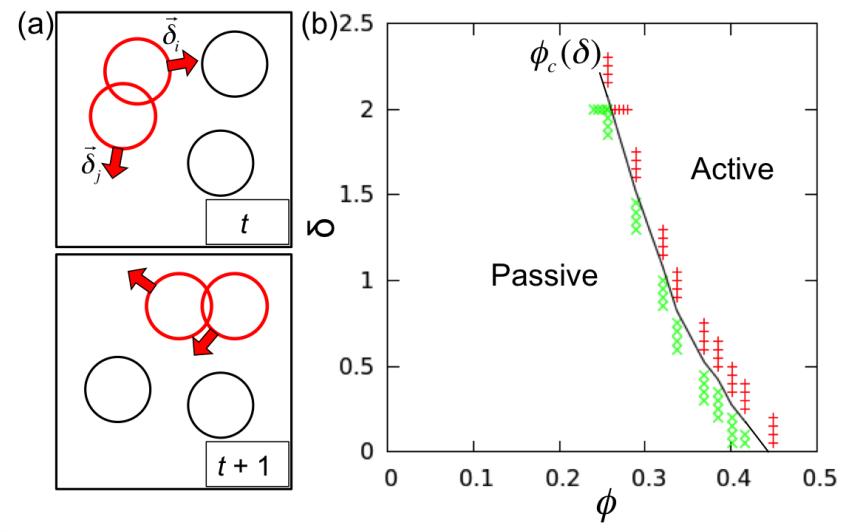

FIG. 1: (a) Sketch of the model: Particles overlaping at time $t$ (red) are simultaneously moved by an independent random amount. Particles with no overlap (black) are immobile, but may become mobile at later time (color online). (b) The $(\phi, \delta)$ phase diagram with a passive region where the number of mobile particles vanishes at long time and an active phase where particle overlaps are constantly destroyed and created. The line of second-order critical points is determined from investigating the state points shown with symbols. 
incremented by one unit. The model has two control parameters: the area fraction $\phi=\frac{\pi N \sigma^{2}}{4 L^{2}}$, and the maximal amplitude of the random kicks $\delta$. We use $\sigma$ as the unit length and we vary the area fraction by changing the number of particles $N$ while keeping the system size fixed at $L=280$ (unless mentioned otherwise).

Our model represents an isotropic version of the periodically sheared system considered in Ref. 4], where random kicks were given to particles virtually colliding with neighbors during a shear deformation cycle (the shear cycle is actually not performed). This original rule is in fact equivalent to giving a random kick to each particle having at least one neighbor in an anisotropic area near its center [21. In our model, we consider that this area is circular, and $\sigma$ represents its diameter. This small simplification makes the determination of the critical properties of the model simpler because it prevents the development of locally anisotropic correlations [22, which could affect the numerical value of the measured exponents, but not the overall qualitative behavior that we report. Our setup is also physically meaningful, as it depicts the experimental situation where a non-Brownian colloidal suspension is driven periodically by a periodic change of the particle diameters leading to irreversible collisions. This is obviously equivalent to isotropic compression cycles of a colloidal system. Such experiments could be realized experimentally using thermosensitive colloidal particles [13].

As expected [4] we find that below a critical density $\phi_{c}(\delta)$, the number of active particles evolves to zero (no more overlap) and all particles stop moving; this corresponds to the "reversible" phase of the experiments with periodic forcing. Above $\phi_{c}(\delta)$, the number of active particles fluctuates at steady state around its mean non-zero value, and the system is diffusive. By carefully exploring the steady state properties [23] of the state points shown in Fig. 1(b), we have numerically determined the critical line $\phi_{c}(\delta)$ separating the two phases. To determine the critical properties of the model, we used the order parameter, which is the fraction of active particles, $f_{a}(t)=N_{a} / N$, where $N_{a}(t)$ is the number of particles having overlaps at time $t$. The spatio-temporal properties of $f_{a}(t)$ display critical properties that can be compared to known universality classes [24]. While such a study is not problematic for computer simulations, it is more difficult in experiments as it requires tracking the displacement of all particles at all times and separating mobile from immobile particles. We provide below simpler observables which exhibit relevant signatures of the underlying phase transition.

A simpler quantity, measured in the original experimental study, is the single particle diffusion constant, defined as $D=\lim _{t \rightarrow \infty}\left\langle|\Delta \vec{r}(t)|^{2}\right\rangle /(4 t)$, where $\Delta \vec{r}(t)$ represents the displacement of a given particle over a time $t$. The brackets indicate an ensemble average (equivalent, in steady state, to a time average). This measurement can be performed using tracer particles followed over long times. In our model, we find that $D=0$ for $\phi<\phi_{c}$, and it emerges continuously above $\phi_{c}$ :

$$
D \sim\left(\phi-\phi_{c}\right)^{\beta}, \quad \phi \rightarrow \phi_{c}^{+} .
$$

We measure $\beta \simeq 0.572$, which is similar to the value found in related studies [4, 11-13, 25. Additionally, we find that $\beta$ remains constant, within statistical uncertainty, along the line $\phi_{c}(\delta)$. For most of this paper, we thus fix $\delta=0.5$ for which $\phi_{c} \simeq 0.375$. The critical exponent $\beta$ in Eq. (1) is relevant because it is directly related to the order parameter, $\left\langle f_{a}\right\rangle$. To see this, let us rewrite the particle displacement as $\Delta \vec{r}(t)=$ $\sum_{t^{\prime}=0}^{t-1}\left[\vec{r}\left(t^{\prime}+1\right)-\vec{r}\left(t^{\prime}\right)\right]$. Denoting by $t_{a}$ the number of timesteps where the tracer is mobile between times 0 and $t$, the displacement is the sum of $t_{a}$ random kicks. As a result, $D$ scales as $t_{a} / t$, which represents the fraction of the time when the tracer is mobile. When $t \rightarrow \infty$, this becomes the ensemble average $\left\langle f_{a}\right\rangle$. In two dimensions, $\beta \approx 0.58$ for directed percolation, and $\beta \approx 0.64$ for conserved directed percolation (or 'Manna' universality class) [2. Our simulations appear closer to the directed percolation universality class.

In experiments with non-Brownian particles, it is easy to visualise particle configurations and analyse static density fluctuations. In Fig. 22(a), we show a snapshot of the system close to criticality, where very few active particles coexist with many passive ones. The structure appears globally homogeneous with no sign of large scale density fluctuations. At smaller scale, particles form short onedimensional clusters, or 'strings', which are disconnected and do not percolate throughout the system. This tendency is confirmed in the radial distribution function [26], $g(r)$, shown in Fig. 2(b) for various densities across the critical point. We see that $g(r)$ has two peaks at $r=1$ and $r=2$, indicative of the string-like structure at short lengthscales. The sharpness of these two peaks is controlled by the amplitude $\delta$ of the random jumps; they become sharper as $\delta \rightarrow 0$. Very similar radial distribution functions have recently been observed in a periodically driven colloidal suspension [16. More importantly, we conclude from Fig. 2(b) that $g(r)$ is rather insensitive to the crossing of the phase transition.

While this might indicate that static density fluctuations are insensitive to the critical point, Fourier transforming $g(r)$ to get the structure factor $S(q)$ shows interesting behaviour, as suggested very recently [27]. In Figs. 2(c,d) we show the low- $q$ behavior of $S(q)$ respectively above and below the critical point. In this log$\log$ representation it is clear that $S(q \rightarrow 0)$ becomes extremely small as $\phi \rightarrow \phi_{c}^{ \pm}$, with emerging power laws. Notice that $S(q)$ converges to the same form on both sides, but convergence from the absorbing phase is slower, as the system retains memory of the disordered initial conditions on very large scale. However, careful analysis of the density evolution [24] reveals that $S(q \rightarrow 0)$ vanishes 
(a)

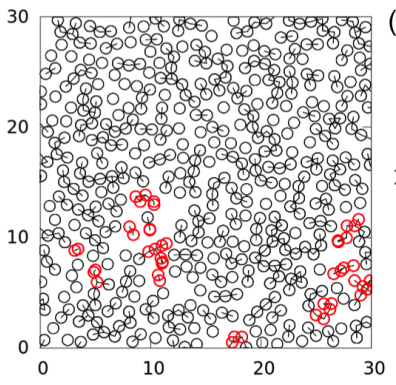

(b)

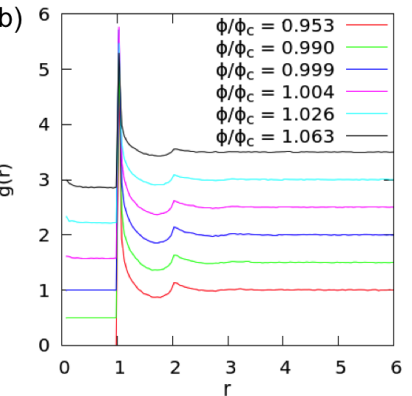

(c)
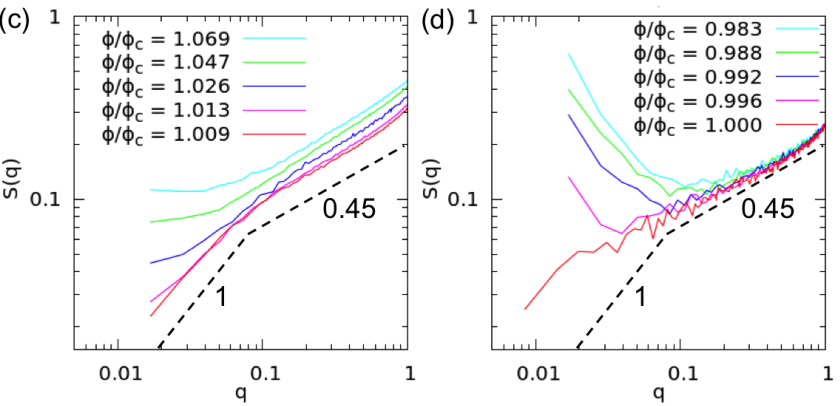

FIG. 2: (a) Typical snapshot close to criticality $\left(\phi / \phi_{c}=\right.$ 1.004 and $\delta=0.05)$ at steady state. Active/passive particles are shown in red/black. Bonds are drawn between particles whose separation is less than 1.05 to reveal string-like clusters. (b) The radial distribution function for different densities (for $\delta=0.05$ ) reveals two peaks at $r=1$ and $r=2$, due to the strings but $g(r)$ does not change significantly across the transition. (c) The structure factor for different densities $\phi>\phi_{c}$ reveals hyperuniformity at large scale close to criticality $\phi_{c} \simeq 0.37499$, with $S(q \rightarrow 0) \sim q$ crossing over to a different power law. (d) The structure factor for $\phi<\phi_{c}$ behaves similarly as $\phi_{c}$ is approached. The system size is $L=280$ except for $\phi / \phi_{c}=1.000$ for which $L=560$ is used.

precisely at $\phi_{c}$, where $S(q) \approx q$ up to $q \approx 0.05$, crossing over to $S(q) \approx q^{0.45}$ at larger $q$. A vanishing $S(q \rightarrow 0)$ physically means that density fluctuations are strongly suppressed at large scale, which is termed 'hyperuniformity' 28. The linear behaviour with $q$ implies that the number of particles in a (large) subsystem of size $L$ obeys $\left\langle\Delta N^{2}\right\rangle /\langle N\rangle \sim L^{-1}$. In an equilibrium fluid, this ratio is instead independent of $L$. Hyperuniformity has been reported in a number of nonequilibrium situations [28 32], among which hard sphere jammed packings. However the critical density here is much smaller than the jamming density and the hyperuniform structure is different from that of compressed hard spheres. A previous study [27] suggested that $S(q) \sim q^{0.45}$ reflects the asymptotic behavior of $S(q)$, whereas we find that this is only a transient. These findings imply nonetheless that static fluctuations reveal a striking signature of criticality, which has not yet been investigated experimentally [3, 13, 16].

We now turn to the dynamics. Close to the irreversibility transition, we detect strong signatures of dynamic heterogeneities, reminiscent of observations in disordered systems approaching dynamic arrest (such as dense col-

loidal suspensions) 20. This analogy is useful, as it provides us with a toolbox to directly reveal the criticality associated to the nonequilibrium phase transition.

A striking observation stems from tracer trajectories, see Figs. 3(a,b). Over short times, Fig. 3(a), the trajectory is characterised by long waiting periods (when the particle is passive) and a few moments where the particle makes several rapid jumps (when the particle is active). Because activity is sparse close to $\phi_{c}$, particles are necessarily immobile most of the time. Such intermittency is also observed in glassy fluids where particles are caged over long periods [33]. Much longer trajectories resemble ordinary Brownian motion, Fig. 3(b), suggesting that Fickian diffusion is recovered at large scale. Intermittent, non-Fickian dynamics thus represents another signature of the criticality, which could be systematically investigated experimentally through the selfintermediate function $F_{s}(q, t)=\left\langle\frac{1}{N} \sum_{i} c_{i}(q, t)\right\rangle$, where $c_{i}(q, t)=\cos \left[\vec{q} \cdot \Delta \vec{r}_{i}(t)\right]$. Physically, $F_{s}(q, t)$ relaxes from 1 to 0 when particles have moved an average distance $\frac{2 \pi}{q}$.

The relaxation time $\tau(q)$ (defined as $F_{s}(q, \tau)=e^{-1}$ ) is plotted in Fig. 3(c). Over large distances $(q \rightarrow 0)$, Fickian behaviour is observed, $\tau(q) \sim 1 /\left(D q^{2}\right)$. On the other hand, at shorter lengthscales $\tau(q)$ crosses over to a plateau value, $\tau_{\infty}(\phi)$. As $\phi \rightarrow \phi_{c}^{+}$, this non-Fickian plateau regime becomes dominant. Physically, $\tau_{\infty}$ represents the typical waiting time before an immobile particle becomes active. We measure $\tau_{\infty} \sim\left(\phi-\phi_{c}\right)^{-\nu_{\|}}$, where $\nu_{\|} \simeq 1.27$, see Fig 3 (f). Interestingly, we found numerically that the same exponent $\nu_{\|}$controls the temporal fluctuations of the order parameter, $f_{a}(t)$ 24]. This is close to the directed percolation value $\nu_{\|}=1.30\left(\nu_{\|}=\right.$ 1.23 for conserved directed percolation) [2]. Finally, because $\tau_{\infty}$ and $D^{-1}$ obey different power laws, we can define a diverging crossover lengthscale for the emergence of Fickian diffusion [33], $\ell_{F} \sim \sqrt{D \tau_{\infty}} \sim\left(\phi-\phi_{c}\right)^{-\left(\nu_{\|}-\beta\right) / 2}$ (see Fig. 3(c)), indicating that diffusion is non-Fickian at all length scale at the critical point.

Intermittency suggests that mobile and immobile particles coexist in space. We now show that the associated dynamic fluctuations also diverge at $\phi_{c}$. To this end, we study spatial correlations of particle displacements, in analogy with measurements in dense fluids 20. We first introduce a 'four-point' structure factor $S_{4}(k, t)$ [20]:

$$
\left.S_{4}(k, t)=\left\langle\frac{1}{N} \sum_{i, j} e^{i \mathbf{k} \cdot\left(\mathbf{r}_{i}-\mathbf{r}_{j}\right)} c_{i}(q, t) c_{j}(q, t)\right)\right\rangle,
$$

where we fix $q=2 \pi$ [in the plateau regime of $\tau(q)$ in Fig 3(c)]. Physically, $S_{4}(k, t)$ measures (in the Fourier domain) spatial correlations between particles which have moved a distance $q^{-1}$ during the time interval $t$. We also define the four-point susceptibility $\chi_{4}(t)=S_{4}(k \rightarrow$ $0, t)$, which measures the variance of spontaneous fluctuations of the time correlation function $F_{s}(q, t)$.

The dynamic susceptibility is plotted in Fig. 3(d) for 

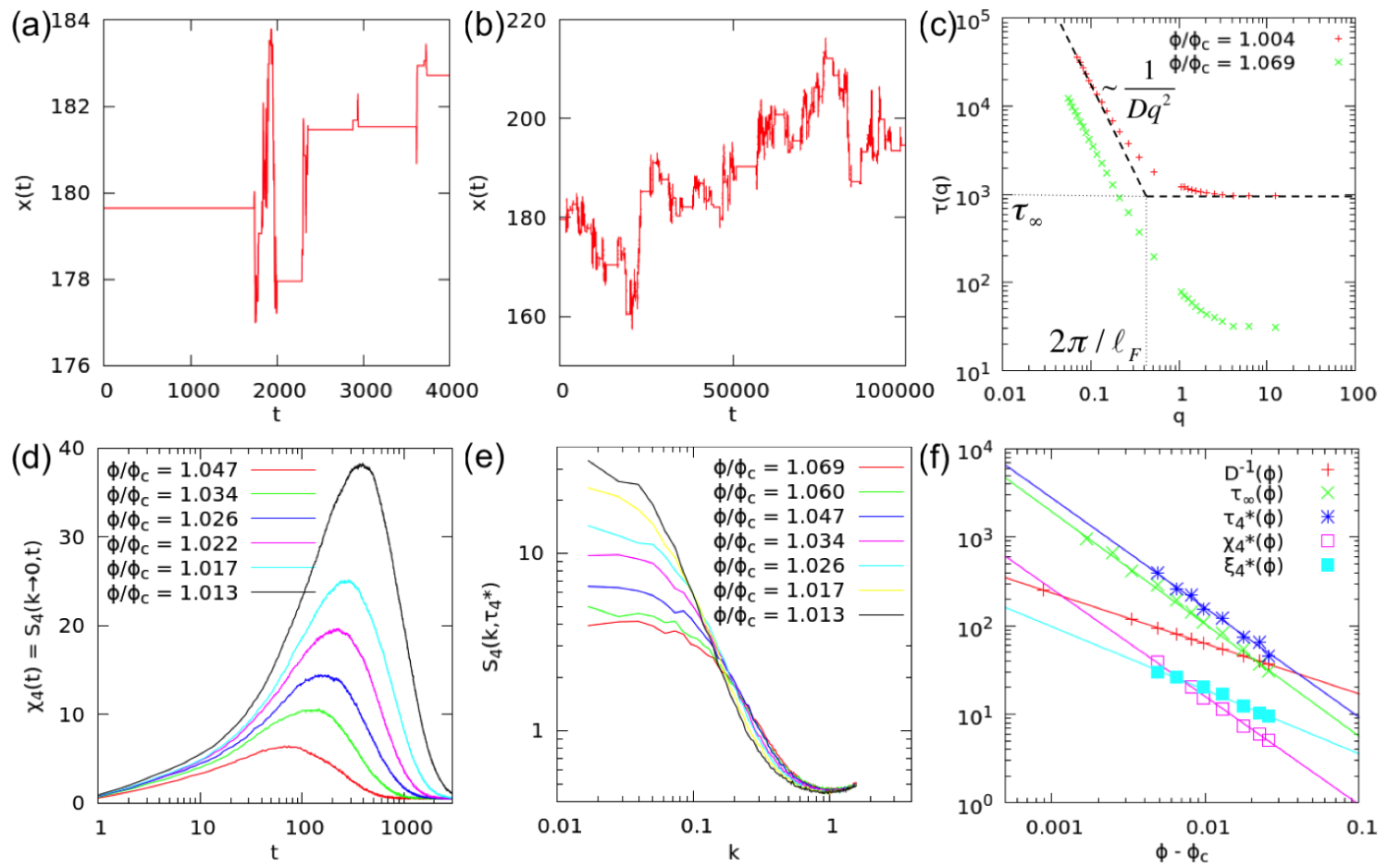

FIG. 3: (a) Typical particle trajectory along the $x$-axis for $\phi / \phi_{c}=1.009$ characterised by long waiting periods (when the particle is passive) and few jumps (when the particle is active). (b) Over a much longer time interval, the same trajectory resembles an ordinary random walk. (c) Wavevector dependence of the relaxation timescale $\tau(q)$ for two different densities. A non-Fickian $\left(\tau \sim \tau_{\infty}(\phi)\right)$ to Fickian $\left(\tau \sim 1 /\left(D q^{2}\right)\right)$ crossover is observed at a wavector $\left(2 \pi / \ell_{F}(\phi)\right)$ which decreases as the transition is approached. (d) The four-point susceptibility $\chi_{4}(t)$ quantifies spatially correlated dynamics over a time interval $t$. It shows a peak at $t=\tau_{4}^{*}(\phi)$ which diverges as $\phi \rightarrow \phi_{c}^{+}$. (e) Four-point structure factor $S_{4}\left(k, t=\tau_{4}^{*}\right)$ as a function of the wavevector $k$ for different densities reveals a diverging dynamic correlation length $\xi_{4}^{*}(\phi)$. (f) Critical power laws for quantities measured in this work: inverse diffusion constant $D^{-1}$ (exponent 0.572), Fickian crossover timescale $\tau_{\infty}(1.27)$, dynamic timescale $\tau_{4}^{*}(1.24)$, maximum susceptibility $\chi_{4}^{*}(1.22)$, and dynamic lengthscale $\xi_{4}^{*}(0.72)$.

different densities. For a given $\phi, \chi_{4}(t)$ exhibits a maximum, $\chi_{4}^{*}$, at a time $\tau_{4}^{*}$. Both $\tau_{4}^{*}$ and $\chi_{4}^{*}$ grow rapidly as $\phi_{c}$ is approached, and obey power laws, see Fig. $3(\mathrm{f})$ : $\tau_{4}^{*} \sim\left(\phi-\phi_{c}\right)^{-\nu_{\|}}$, with $\nu_{\|} \simeq 1.24$, compatible with the result for $\tau_{\infty}$. Similarly, $\chi_{4}^{*} \sim\left(\phi-\phi_{c}\right)^{-\gamma}$, with $\gamma \simeq 1.22$. The divergence of $\chi_{4}^{*}$ is accompanied by a diverging correlation length, as revealed by the evolution of $S_{4}(k, t)$ in Fig. 3(e). Here we fix $t=\tau_{4}^{*}$ when the correlation is maximal. We observe a growing peak at low wavevector shifting to lower $k$ as $\phi$ increases. We follow established procedures [20] and extract the dynamic lengthscale $\xi_{4}^{*}$ by using the following scaling form: $S_{4}\left(k, \tau_{4}^{*}\right) / \chi_{4}^{*}=F\left(k \xi_{4}^{*}(\phi)\right)$, where $F(x)$ is a scaling function independent of $\phi$. As shown in Fig. 3(f), $\xi_{4}^{*}$ obeys a power law divergence, $\xi_{4}^{*} \sim\left(\phi-\phi_{c}\right)^{\nu_{\perp}}$. We measure $\nu_{\perp} \simeq 0.72$. We found numerically that a similar critical exponent controls the divergence of the order parameter correlation length 24. Again, our measurements compare well to the directed percolation exponent, $\nu_{\perp}=0.72$ $\left(\nu_{\perp}=0.80\right.$ for conserved directed percolation) [2].

Our results demonstrate that the irreversibility transition observed in periodically driven systems has interesting qualitative analogies with glassy systems. In both cases, the radial distribution function $g(r)$ appears insensitive to dynamic arrest, whereas other quantities display stronger signatures. We have reported a strong suppression of the density fluctuations at large scales, and a divergence of several dynamic quantities associated to single particle and collective dynamics. The analogy between the two types of systems suggests that particlebased measurements and observables developed for glassy materials could prove useful in driven suspensions. These tools could in particular reveal whether the 'singularityfree' explanation based on the Lyapunov instability is experimentally relevant. Our work also suggests that it would be interesting to characterize more precisely the static structure and dynamic correlations in the vicinity of the yielding transition in dense suspensions under oscillatory shear [7, 13, 17, 25, 34, which represents another important situation where a reversibility transition and a transition to chaos [35] should be better understood.

We thank D. Bartolo and R. Jack for useful discussions. The research leading to these results has received funding from the European Research Council under the European Unions (EU) Seventh Framework Programme (FP7/2007-2013)/ERC Grant Agreement No. 306845. 
[1] H. Hinrichsen, Nonequilibrium Critical Phenomena and Phase Transitions into Absorbing States, Adv. Phys. 49 (2000) 49.

[2] S. Lubeck, Universal scaling behaviour of nonequilibrium phase-transitions, Int. J. Mod. Phys. B 18 (2004) 3977.

[3] D. J. Pine, J. P. Gollub, J. F. Brady, and A. M. Leshansky, Chaos and irreversibility in sheared suspensions, $\mathrm{Na}$ ture 438 (2005) 997.

[4] L. Corte, P. M. Chaikin, J. P. Gollub, and D. J. Pine, Random organization in periodically driven systems, $\mathrm{Na}$ ture Physics 4 (2008) 420.

[5] L. Corte, S. J. Gerbode, W. Man, and D. J. Pine, SelfOrganized Criticality in Sheared Suspensions, Phys. Rev. Lett. 103 (2009) 248301.

[6] J. S. Guasto, A. S. Ross, and J. P. Gollub, Hydrodynamic irreversibility in particle suspensions with nonuniform strain, Phys. Rev. E 81 (2010) 061401.

[7] J. D. Paulsen, N. C. Keim, and S. R. Nagel, Multiple Transient Memories in Experiments on Sheared Non-Brownian Suspensions, Phys. Rev. Lett. 113 (2014) 068301.

[8] N. C. Keim, and P. E. Arratia, Mechanical and Microscopic Properties of the Reversible Plastic Regime in a 2D Jammed Material, Phys. Rev. Lett. 112 (2014) 028302.

[9] A. Franceschini, E. Filippidi, E. Guazzelli, and D. J. Pine, Dynamics of non-Brownian fiber suspensions under periodic shear, Soft Matter 10 (2014) 6722.

[10] G. I. Menon and S. Ramaswamy, Universality Class of the Reversible-Irreversible Transition in Sheared Suspensions, Phys. Rev. E 79 (2009) 061108.

[11] N. Mangan, C. Reichhardt, and C. J. Olson Reichhardt, Reversible to Irreversible Flow Transition in Periodically Driven Vortices, Phys. Rev. Lett. 100 (2008) 187002.

[12] C. Reichhardt, and C. J. Olson Reichhardt, Random Organization and Plastic Depinning, Phys. Rev. Lett. 103 (2009) 168301.

[13] K. H. Nagamanasa, S. Gokhale, A. K. Sood, and R. Ganapathy, Experimental signatures of a nonequilibrium phase transition governing the yielding of a soft glass, Phys. Rev. E 89 (2014) 062308.

[14] G. During, D. Bartolo, and J. Kurchan, Irreversibility and self-organisation in hydrodynamic echo experiments, Phys. Rev. E 79 (2009) 030101.

[15] B. Metzger and J. E. Butler, Irreversibility and chaos: Role of long-range hydrodynamic interactions in sheared suspensions, Phys. Rev. E 82 (2010) 051406.

[16] R. Jeanneret and D. Bartolo, Geometrically protected reversibility in hydrodynamic Loschmidt-echo experiments, Nature Comm. 5 (2014) 3474.

[17] I. Regev, T. Lookman, and C. Reichhardt, Onset of irreversibility and chaos in amorphous solids under periodic shear, Phys. Rev. E 88 (2013) 062401.

[18] P. Sundararajan, J. D. Kirtland, D. L. Koch, and A. D. Stroock, Impact of chaos and Brownian diffusion on irreversibility in Stokes flows, Phys. Rev. E 86 (2012) 046203.

[19] B. Metzger, P. Pham, and J. E. Butler, Irreversibility and chaos: Role of lubrication interactions in sheared suspensions, Phys. Rev. E 87 (2013) 052304.

[20] Dynamical heterogeneities in glasses, colloids and granular materials, Eds: L. Berthier, G. Biroli, J.-P. Bouchaud, L. Cipelletti, and W. van Saarloos (Oxford University Press, Oxford, 2011).

[21] L. Milz and M. Schmiedeberg, Connecting the random organization transition and jamming within a unifying model system, Phys. Rev. E 88 (2013) 062308.

[22] D. V. Denisov, M. T. Dang, B. Struth, G. H. Wegdam, and P. Schall, Particle response during the yielding transition of colloidal glasses, arXiv:1401.2106 1 .

[23] For a given $\phi<\phi_{c}$, we run several simulations from different initial random configurations and wait until each simulation reaches an absorbing state. Static quantities are then ensemble-averaged over different runs. On the other hand for $\phi>\phi_{c}$, long time average is sufficient since the system is ergodic.

[24] E. Tjhung and L. Berthier, in preparation.

[25] D. Fiocco, G. Foffi, and S. Sastry, Oscillatory athermal quasistatic deformation of a model glass, Phys. Rev. E 88 (2013) 020301(R).

[26] J. P. Hansen and I. R. McDonald, Theory of Simple Liquids (Elsevier, Amsterdam, 1986).

[27] D. Hexner and D. Levine, Hyperuniformity of critical absorbing states, Phys. Rev. Lett. 114, (2015) 110602.

[28] S. Torquato and F. H. Stillinger, Local density fluctuations, hyperuniformity, and order metrics, Phys. Rev. E 68 (2003) 041113.

[29] A. Donev, F. H. Stillinger, and S. Torquato, Unexpected fluctuations in jammed disordered sphere packings, Phys. Rev. Lett. 95 (2005) 090604.

[30] L. Berthier, P. Chaudhuri, C. Coulais, O. Dauchot, and P. Sollich, Suppressed compressibility at large scale in jammed packings of size-disperse spheres, Phys. Rev. Lett. 106 (2011) 120601.

[31] Y. Jiao, T. Lau, H. Haztzikirou, M. Meyer-Hermann, J. C. Corbo, and S. Torquato, Avian Photoreceptor Patterns Represent a Disordered Hyperuniform Solution to a Multiscale Packing Problem, Phys. Rev. E 89 (2004) 022721.

[32] R. L. Jack, I. R. Thompson, and P. Sollich, Hyperuniformity and phase separation in biased ensembles of trajectories for diffusive systems, Phys. Rev. Lett. 114 (2015) 060601.

[33] L. Berthier, D. Chandler, and J. P. Garrahan, Length scale for the onset of Fickian diffusion in supercooled liquids, Europhys. Lett. 71 (2005) 320.

[34] E. D. Knowlton, D. J. Pine, and L. Cipelletti, A microscopic view of the yielding transition in concentrated emulsions, Soft Matter 10 (2014) 6931.

[35] E. J. Banigan, M. K. Illich, D. J. Stace-Naughton, and D. A. Egolf, The chaotic dynamics of jamming, Nature Phys. 9 (2013) 288. 